\title{
Effect of a cosmological constant on propagation of vacuum polarized photons in stationary spacetimes
}

\author{
Sourav Bhattacharya ${ }^{\mathrm{a}}$ \\ Department of Physics, Institute for Theoretical and Computational Physics, University of Crete, 70013 Heraklion, Greece
}

Received: 18 May 2015 / Accepted: 24 May 2015 / Published online: 3 June 2015

(C) The Author(s) 2015. This article is published with open access at Springerlink.com

\begin{abstract}
Consideration of vacuum polarization in quantum electrodynamics may affect the momentum dispersion relation for photons for a non-trivial background, due to the appearance of curvature dependent terms in the effective action. We investigate the effect of a positive cosmological constant $\Lambda$ on this at one-loop order for stationary $\Lambda$-vacuum spacetimes. To the best of our knowledge, so far it only has been shown that $\Lambda$ affects the propagation in a time dependent black hole spacetime. Here we consider the static de Sitter cosmic string and the Kerr-de Sitter spacetime to show that there might occur a non-vanishing effect due to $\Lambda$ for physical polarizations. The consistency of these results with the polarization sum rule is discussed.
\end{abstract}

\section{Introduction}

If we consider quantum electrodynamics in curved spacetime, the effective action for the photon contains, from the loop integrals, some finite part which depends on both the field strength and the background curvature. Such finite parts originate from the curvature dependence of the propagator in curved spacetime. In that case the photon equation of motion gets modified and consequently the dispersion relation for null geodesics may no longer hold due to the gravitational tidal forces, as was first shown in [1].

Since then a lot of attention has been given to this exotic effect [2-14]. Such an effect may also be present for massless spin-1/2 fields [15], gravitons [16], and even in flat spacetimes with non-trivial vacuum, e.g. $[17,18]$.

The velocity shifts of photons were investigated in various black hole backgrounds $[3,5,7,8]$. For various aspects associated with this phenomenon including the preservation of unitarity and causality, we refer the reader to $[2,6,9]$.

The chief concern of this work is to investigate the role of a positive cosmological constant $\Lambda$ in this phenomenon.

\footnotetext{
a e-mail: souravbhatta@physics.uoc.gr
}

The physical motivation of this study comes from the observation of accelerated expansion of our universe $[19,20]$. It was shown in [1] that there is no modification of the photon's speed in any maximally symmetric space like de Sitter space. In [8] various stationary and time dependent black holes with $\Lambda$ were considered and it was shown that although the velocity of the vacuum polarized photons gets modified, $\Lambda$ contributes to this only in the time dependent case. Therefore, it is interesting to see whether we may find some nontrivial effect due to $\Lambda$ in some physically interesting stationary spacetimes.

In particular, we shall consider below two $\Lambda>0$ spacetimes - the Linet-Tian cosmic string [21,22] and the Kerr-de Sitter [23] to show that we may have non-vanishing effects due to $\Lambda$, for physical or transverse polarizations.

For the cosmic string spacetime in particular, the false vacuum of the scalar field inside the string core makes the effective value of the cosmological constant higher and hence for such spacetimes this effect may be considerable inside the string core.

\section{Calculation of the velocity shifts}

The basic calculational scheme uses the one-loop corrected effective equation of motion for photons in a weakly curved space for QED [1,6,8],

$$
\begin{gathered}
\nabla_{a} F^{a b}+\frac{2}{m_{\mathrm{e}}^{2}} \nabla_{a}\left[2 a R F^{a b}+b\left(R_{c}^{a} F^{c b}-R_{c}^{b} F^{c a}\right)\right] \\
+\frac{4 c}{m_{\mathrm{e}}^{2}} \nabla_{a}\left(R^{a b}{ }_{c d} F^{c d}\right)=0
\end{gathered}
$$

where $m_{\mathrm{e}}$ is the electron mass and $a=-\frac{5 \alpha}{720 \pi}, b=\frac{26 \alpha}{720 \pi}$, and $c=-\frac{2 \alpha}{720 \pi} ; \alpha$ is the fine structure constant. Using the eikonal approximation Eq. (1) can be cast in the leading order [1] form, 
$k^{2} a_{b}+\frac{2 b}{m_{\mathrm{e}}^{2}} R_{a c}\left(k^{a} k^{c} a_{b}-k^{a} a^{c} k_{b}\right)+\frac{8 c}{m_{\mathrm{e}}^{2}} R_{a b c d} k^{a} k^{c} a^{d}=0$,

where $k^{a}$ is the photon momentum, $k^{2}=k_{a} k^{a}$, and $a_{b}$ is the polarization vector, $a \cdot k=0$. We put above $R_{a b}=\Lambda g_{a b}$ for $\Lambda$-vacuum Einstein spaces. The above equation is the leading departure from the null geodesic approximation. We may note here that if we assume the spacetime to be maximally symmetric de Sitter, then the Riemann tensor gets decomposed into terms proportional to $\Lambda$ and $g_{a b}$, and accordingly we get $k^{2}=0$.

Precisely, our goal here is to provide examples of nonmaximally symmetric stationary $\Lambda$-vacuum Einstein spaces for which not only $k^{2} \neq 0$, but also $\Lambda$ has non-vanishing contributions into this, by solving Eq. (2) for physical or transverse polarizations.

\subsection{The Linet-Tian-cosmic string spacetime}

Let us start with the static cylindrical Linet-Tian or NielsenOlesen cosmic string spacetimes [21,22], the exterior of which is given by

$$
\begin{aligned}
\mathrm{d} s^{2}= & \cos ^{\frac{4}{3}} \frac{\rho \sqrt{3 \Lambda}}{2}\left(\mathrm{~d} t^{2}-\mathrm{d} z^{2}\right) \\
& -\mathrm{d} \rho^{2}-\frac{4 \delta^{2}}{3 \Lambda} \sin ^{2} \frac{\rho \sqrt{3 \Lambda}}{2} \cos ^{-\frac{2}{3}} \frac{\rho \sqrt{3 \Lambda}}{2} \mathrm{~d} \phi^{2},
\end{aligned}
$$

where $\delta$ is a constant representing the conical singularity. The inside core metric for the Nielsen-Olesen configuration near the axis looks formally the same as above, with $\delta=1$, and $\Lambda$ replaced with an effective cosmological constant $\Lambda^{\prime}=\Lambda+2 \pi \lambda \eta^{4}$ [22], where $\lambda$ and $\eta$ are, respectively, the coupling constant for a quartic self interaction and the expectation value of the complex scalar field in its false vacuum. The above spacetime has a curvature singularity at $\rho=\frac{\pi}{\sqrt{3 \Lambda}}[21]$. Since Eq. (2) is valid only in the weak curvature limit, the following calculations will not hold near that singularity. Clearly, the above metric globally cannot represent a physical spacetime itself, and it has to be embedded within some other spacetime to get a reasonable global scenario. In any case, the Linet-Tian spacetime can be a very good realistic model for spacetimes with a positive cosmological constant in the vicinity of a cylindrical mass distribution of compact cross section.

We choose an orthonormal basis: $\omega^{0}=\sqrt{g_{t t}} \mathrm{~d} t, \omega^{1}=$ $\sqrt{-g_{\phi \phi}} \mathrm{d} \phi, \omega^{2}=\mathrm{d} \rho, \omega^{3}=\sqrt{-g_{z z}} \mathrm{~d} z$. Following e.g. [8], we next define the tensors $U_{a b}^{\mu v}=\delta_{[a}^{\mu} \delta_{b]}^{v}$. With the help of these tensors, we express the components of the Riemann tensor by (see e.g. Chapter 6 of [24], for expressions in generic stationary axisymmetric spacetimes)

$$
\begin{aligned}
R_{a b c d}= & E\left(U_{a b}^{12} U_{c d}^{12}-U_{a b}^{30} U_{c d}^{30}\right) \\
& +F\left(U_{a b}^{10} U_{c d}^{10}+U_{a b}^{20} U_{c d}^{20}-U_{a b}^{23} U_{c d}^{23}-U_{a b}^{31} U_{c d}^{31}\right)
\end{aligned}
$$

where $E(\rho)=\frac{\Lambda}{3} \tan ^{2}\left(\frac{\rho \sqrt{3 \Lambda}}{2}\right)$, and $F(\rho)=\frac{\Lambda}{2}\left(1+\frac{1}{3} \tan ^{2}\right.$ $\left.\left(\frac{\rho \sqrt{3 \Lambda}}{2}\right)\right)$, so that $R_{1010}=F(\rho)$, and so on.

Since the conical singularity term $\delta$ in Eq. (3) does not contribute to the curvature, it is clear that the above expressions remain formally unchanged near the axis of the string, with $\Lambda$ replaced with $\Lambda^{\prime}=\Lambda+2 \pi \lambda \eta^{4}$.

Also, we define the individual momenta through $U_{a b}^{\mu \nu}$ : $k^{b} U_{a b}^{\mu \nu}$,

$$
\begin{aligned}
l_{a}= & \left(k_{0} \delta_{a}^{1}+k_{1} \delta_{a}^{0}\right), \quad m_{a}=\left(k_{0} \delta_{a}^{2}+k_{2} \delta_{a}^{0}\right), \\
& n_{a}=\left(k_{0} \delta_{a}^{3}+k_{3} \delta_{a}^{0}\right), \\
p_{a}= & \left(k_{1} \delta_{a}^{2}-k_{2} \delta_{a}^{1}\right), \quad q_{a}=\left(k_{2} \delta_{a}^{3}-k_{3} \delta_{a}^{2}\right), \\
& r_{a}=\left(k_{3} \delta_{a}^{1}-k_{1} \delta_{a}^{3}\right) .
\end{aligned}
$$

We contract Eq. (2) with a vector field $v^{b}$, where $v^{b}$ at a time corresponds to any one of the above momenta, and using Eqs. (4) and (5) we find

$$
\begin{aligned}
& {\left[1+\frac{2 b \Lambda}{m_{\mathrm{e}}^{2}}\right] k^{2}(v \cdot a)-\frac{8 c \Lambda E}{m_{\mathrm{e}}^{2}}[(v \cdot p)(a \cdot p)-(v \cdot n)(a \cdot n)]} \\
& -\frac{8 c \Lambda F}{m_{\mathrm{e}}^{2}}[(v \cdot l)(a \cdot l)+(v \cdot m)(a \cdot m)-(v \cdot q)(a \cdot q) \\
& -(v \cdot r)(a \cdot r)]=0 .
\end{aligned}
$$

We start by considering the radial photon motion, $k \equiv$ $\left\{k_{0}, 0, k_{2}, 0\right\}$ in Eq. (5) and $k^{2}=k_{0}^{2}-k_{2}^{2}=-m^{2}$. The two physical polarization vectors for radial photons correspond to the azimuthal and axial directions $(a \sim l, n)$.

For radial photons we have $l \equiv\left\{0, k_{0}, 0,0\right\}$ in Eq. (5). First we take $v=l$ in Eq. (6). Also we have in this case $p=-\frac{k_{2}}{k_{0}} l$ and $l \cdot m=l \cdot n=l \cdot q=l \cdot r=0$. Putting all this together, we get

$k^{2}(a \cdot l)\left(1+\frac{2 b \Lambda}{m_{\mathrm{e}}^{2}}\right)+\frac{8 c}{m_{\mathrm{e}}^{2}}\left[E k_{2}^{2}+F k_{0}^{2}\right](a \cdot l)=0$,

with no mixing term $a \cdot n$ with the other physical polarization vector. Putting in this result the expressions of $E$ and $F$ [see after Eq. (4)], we get, since $a \cdot l \neq 0$,

$$
\left|\frac{k_{0}}{k_{2}}\right|_{\phi}=\left(1-\frac{2 c \Lambda}{m_{\mathrm{e}}^{2}} \sec ^{2}\left(\frac{\rho \sqrt{3 \Lambda}}{2}\right)\right) \text {, }
$$

which clearly shows that the null geodesic dispersion relation is violated due to $\Lambda$ via a quantum effect, and since $c=$ $-\frac{2 \alpha}{720 \pi}$ it is superluminal. 
In order to get the equation corresponding to the other polarization vector $(a \sim n)$, we take $v=n$ in Eq. (6). We note from (5) that $q=\frac{k_{2}}{k_{0}} n$ and $n \cdot l=n \cdot m=n \cdot p=n \cdot r=0$. Putting all this together, we solve Eq. (6) as earlier, to get

$$
\left|\frac{k_{0}}{k_{2}}\right|_{z}=\left(1+\frac{2 c \Lambda}{m_{\mathrm{e}}^{2}} \sec ^{2}\left(\frac{\rho \sqrt{3 \Lambda}}{2}\right)\right),
$$

which is subluminal.

Next, let us consider the azimuthal or orbital photons, $k \equiv\left\{k_{0}, k_{1}, 0,0\right\}$, and $k^{2}=k_{0}^{2}-k_{1}^{2}=-l^{2}$, from Eq. (5). The two physical polarization states correspond to the radial and the axial directions, $a \sim m, n$.

We first take $v=m$ in Eq. (6). We have $m \cdot l=m \cdot n=$ $m \cdot q=m \cdot r=0$, and $p=\frac{k_{1}}{k_{0}} m$. Then proceeding as earlier, from Eq. (6) we find

$$
\left|\frac{k_{0}}{k_{1}}\right|_{\rho}=\left(1-\frac{2 c \Lambda}{m_{\mathrm{e}}^{2}} \sec ^{2}\left(\frac{\rho \sqrt{3 \Lambda}}{2}\right)\right) .
$$

Likewise, taking $v=n$ and $r=-\frac{k_{1}}{k_{0}} n$, and noting that the inner products of $n$ with the vectors $(l, m, p, q)$ vanish, Eq. (6) gives

$$
\left|\frac{k_{0}}{k_{1}}\right|_{z}=\left(1+\frac{2 c \Lambda}{m_{\mathrm{e}}^{2}} \sec ^{2}\left(\frac{\rho \sqrt{3 \Lambda}}{2}\right)\right) .
$$

Finally, we come to the photons moving in the axial or $z$ directions, $k \equiv\left\{k_{0}, 0,0, k_{3}\right\}$, and $k^{2}=k_{0}^{2}-k_{3}^{2}=-n^{2}$, in Eq. (5). For physical polarization along the radial direction, we have $a \cdot m \neq 0$ and $q=-\frac{k_{3}}{k_{0}} m$. Then choosing $v=$ $m$ in Eq. (6), and noting $m \cdot p=m \cdot n=m \cdot l=m$. $r=0$, we proceed as earlier to get $k^{2}=0$, i.e. the null geodesic dispersion relation. For polarization along $\phi$, we choose $v=l$, also giving $k^{2}=0$. This fact can be understood as the maximal symmetry of the ' $t-z$ ' plane of the Linet-Tian spacetime (3).

For a Nielsen-Olesen cosmic string spacetime, as we mentioned earlier, sufficiently near the axis, $\Lambda$ can be replaced with $\Lambda^{\prime}=\Lambda+2 \pi \lambda \eta^{4}$, which is due to the false vacuum of the complex scalar field. Then sufficiently inside the core, the non-trivial results of Eqs. (8-11) correspond to replacing $\Lambda$ with $\Lambda^{\prime}$.

\subsection{The Kerr-de Sitter spacetime}

Let us now address the Kerr-de Sitter spacetime [23], which reads in Boyer-Lindquist coordinates

$$
\begin{aligned}
\mathrm{d} s^{2}= & -\Sigma\left(\frac{\mathrm{d} r^{2}}{\Delta_{r}}+\frac{\mathrm{d} \theta^{2}}{R}\right)-\frac{R \sin ^{2} \theta}{\Sigma}\left(\frac{a \mathrm{~d} t-\left(r^{2}+a^{2}\right) \mathrm{d} \phi}{1+\frac{\Lambda a^{2}}{3}}\right)^{2} \\
& +\frac{\Delta_{r}}{\Sigma}\left(\frac{\mathrm{d} t-a \sin ^{2} \theta \mathrm{d} \phi}{1+\frac{\Lambda a^{2}}{3}}\right)^{2},
\end{aligned}
$$

where $\Sigma=r^{2}+a^{2} \cos ^{2} \theta, R=1+\frac{\Lambda a^{2} \cos ^{2} \theta}{3}$ and $\Delta_{r}=$ $\left(r^{2}+a^{2}\right)\left(1-\frac{\Lambda r^{2}}{3}\right)-2 M r$. We shall work in a region far away from the Schwarzschild radius, where we only retain terms linear in the rotation parameter, $a$. Also, owing to the observed tiny value of $\Lambda \sim 10^{-52} \mathrm{~m}^{-2}$, we always have $M \sqrt{\Lambda} \ll 1$, even with large masses such as $M \sim 10^{15} M_{\odot}$. Then, even for $a \sim M$, we can safely ignore $\Lambda a^{2}$ terms for practical purposes. This offers a much simplified form of the above metric,

$\mathrm{d} s^{2} \approx f \mathrm{~d} t^{2}-f^{-1} \mathrm{~d} r^{2}-r^{2} \mathrm{~d} \Omega^{2}+2 \omega r^{2} \sin ^{2} \theta \mathrm{d} t \mathrm{~d} \phi+\mathcal{O}\left(a^{2}\right)$,

where $f(r)=\left(1-\frac{2 M}{r}-\frac{\Lambda r^{2}}{3}\right)$ and $\omega(r)=a\left(\frac{2 M}{r^{3}}+\frac{\Lambda}{3}\right)$. This can be thought of as the leading axisymmetric deformation of the Schwarzschild-de Sitter spacetime due to rotation. Since the diagonal components of the metric represent norms (i.e. scalars) of various coordinate vector fields, they must be even in the rotation parameter, because otherwise they would depend on the sign of $a$, i.e. the direction of rotation of the source and thus we have the above simplification in the leading order. We also note that the effect of $\Lambda$ should be higher as we move away from the gravitating source. In this case, keeping only terms linear in the rotation parameter seems a reasonable approximation, as far as we are interested in the qualitative new effects due to $\Lambda$. On the other hand, if we assume that $a \ll M$, Eq. (13) holds throughout.

We shall see below that such breaking of the spherical symmetry leads to a non-vanishing contribution of $\Lambda$ in the photon propagation.

Following [24], we define the orthonormal basis $\omega^{0}=$ $\sqrt{f} \mathrm{~d} t, \omega^{1}=r \sin \theta(\mathrm{d} \phi-\omega \mathrm{d} t), \omega^{2}=f^{-\frac{1}{2}} \mathrm{~d} r$, and $\omega^{3}=$ $r \mathrm{~d} \theta$, and compute the components of the Riemann tensor up to terms linear in $a$,

$$
\begin{aligned}
-R_{0101} & =-R_{0303}=R_{1212}=R_{2323}=\frac{M}{r^{3}}-\frac{\Lambda}{3}, \\
R_{0202} & =-R_{1313}=\frac{2 M}{r^{3}}+\frac{\Lambda}{3} \\
R_{1303} & =-R_{1202}=\frac{3 M a \sin \theta}{r^{4}} \sqrt{f} \\
R_{1230} & =R_{1023}=-\frac{1}{2} R_{1302}=-\frac{3 M a \cos \theta}{r^{4}} .
\end{aligned}
$$

Let us first consider the photon motion on the equatorial plane, $\theta=\pi / 2$. We express the non-vanishing curvature components in (14) as earlier by 


$$
\begin{aligned}
R_{a b c d}= & A\left(U_{a b}^{12} U_{c d}^{12}+U_{a b}^{23} U_{c d}^{23}-U_{a b}^{10} U_{c d}^{10}-U_{a b}^{30} U_{c d}^{30}\right) \\
& +B\left(U_{a b}^{20} U_{c d}^{20}-U_{a b}^{31} U_{c d}^{31}\right) \\
& +C\left(U_{a b}^{31} U_{c d}^{30}+U_{a b}^{30} U_{c d}^{31}+U_{a b}^{12} U_{c d}^{20}+U_{a b}^{20} U_{c d}^{21}\right),
\end{aligned}
$$

where $A=\frac{M}{r^{3}}-\frac{\Lambda}{3}, B=\frac{2 M}{r^{3}}+\frac{\Lambda}{3}$, and $C=\frac{3 M a \sqrt{f}}{r^{4}}$. We substitute Eq. (15) into Eq. (2) and contract as earlier with $v^{b}$ to get

$$
\begin{aligned}
& k^{2}\left(1+\frac{2 b \Lambda}{m_{\mathrm{e}}^{2}}\right)(a \cdot v)+\frac{8 c A}{m_{\mathrm{e}}^{2}}[(p \cdot v)(p \cdot a)+(q \cdot v)(q \cdot a) \\
& \quad-(l \cdot v)(l \cdot a)-(n \cdot v)(n \cdot a)] \\
& \quad+\frac{8 c B}{m_{\mathrm{e}}^{2}}[(m \cdot v)(m \cdot a)-(r \cdot v)(r \cdot a)] \\
& \quad+\frac{8 c C}{m_{\mathrm{e}}^{2}}[(r \cdot v)(n \cdot a)(r \cdot a)(n \cdot v) \\
& \quad+(p \cdot v)(m \cdot a)+(p \cdot a)(m \cdot v)]=0
\end{aligned}
$$

where the vectors $l, m, n$ etc. are defined in Eq. (5).

Let us start by considering the radial photons, $k \equiv$ $\left\{k_{0}, 0, k_{2}, 0\right\}$ and $k^{2}=-m^{2}$ in Eq. (5). One of the two physical polarizations is along the orthonormal basis $\omega^{1}$, defined above Eq. (14). In this case $a \cdot l \neq 0$. The other physical polarization is along the polar direction, $a \sim n$.

First we take $v=l$ in Eq. (16). Also, for radial photons we have from Eq. (5), $p=-\frac{k_{2}}{k_{0}} l$, and $l \cdot q=l \cdot n=l \cdot m=$ $l \cdot r=0$. Then it turns out that

$k^{2}\left(1+\frac{2 b \Lambda}{m_{\mathrm{e}}^{2}}\right)+\frac{8 c A}{m_{\mathrm{e}}^{2}}[(p \cdot l)(a \cdot p)-(l \cdot l)(a \cdot l)]=0$.

We have for radial photons, $l^{2}=-k_{0}^{2}$, and $(p \cdot l)(p \cdot a)=$ $-k_{2}^{2}(a \cdot l)$. Putting this back into Eq. (17), we get $k^{2}=0$.

Likewise, we next take $v=n$ in Eq. (16). We also have $q=\frac{k_{2}}{k_{0}} n$ in Eq. (5). Proceeding as above we get $k^{2}=0$, as well. Thus for radial photons there are no velocity shifts for physical polarizations.

We note that retaining the generic rotation in the Kerr case gives a velocity shift of at least $\mathcal{O}\left(a^{2}\right)$ [5]. Since we are working only with terms linear in the rotation parameter, we have $k^{2}=0$, analogous to the static spacetimes [8].

Let us now consider the orbital photons. We have $k \equiv$ $\left\{k_{0}, k_{1}, 0,0\right\}, k^{2}=-l^{2}$ in Eq. (5). The two physical polarization vectors correspond to radial and polar directions, $a \sim m, n$.

Taking $v=m$ in Eq. (16) and $p=\frac{k_{1}}{k_{0}} m$ [which follows from (5), for orbital photons], using $m \cdot m=-k_{0}^{2}$ and $p \cdot m=$ $-k_{0} k_{1}$, Eq. (16) reduces to $k^{2}\left(1+\frac{2 b \Lambda}{m_{\mathrm{e}}^{2}}\right)-\frac{8 c}{m_{\mathrm{e}}^{2}}\left[A k_{1}^{2}+B k_{0}^{2}+2 C k_{0} k_{1}\right]=0$.

Since we are working at one-loop order, whenever the momenta are multiplied with the coefficients $b$ or $c$, we can take the null geodesic dispersion relation. This means that, for the last term in the above equation, we can take $k_{0}= \pm k_{1}$, where $+(-)$ sign corresponds to prograde (retrograde) orbits, respectively.

Then we find using the explicit expressions for $A, B$, and $C$ [see below Eq. (15)],

$$
\left|\frac{k_{0}}{k_{1}}\right|_{r}=1+\frac{12 c M}{m_{\mathrm{e}}^{2} r^{3}}\left[1 \pm \frac{2 a}{r}\left(1-\frac{2 M}{r}-\frac{\Lambda r^{2}}{3}\right)^{\frac{1}{2}}\right],
$$

where the $+(-)$ sign corresponds to prograde (retrograde) orbits, respectively.

Similarly for the other physical polarization, setting $v=n$ in Eq. (16), and using $r=-\frac{k_{1}}{k_{0}} n$ [which follows from (5), for this case] yields

$$
\left|\frac{k_{0}}{k_{1}}\right|_{\theta}=1-\frac{12 c M}{m_{\mathrm{e}}^{2} r^{3}}\left[1 \pm \frac{2 a}{r}\left(1-\frac{2 M}{r}-\frac{\Lambda r^{2}}{3}\right)^{\frac{1}{2}}\right] .
$$

This completes the calculation part for the velocity shifts on the equatorial plane.

We note that if we set $M=0$ in Eqs. (19) and (20), we recover the vanishing result of the empty de Sitter space [1].

Let us now check the consistency of our results with the sum rule over the physical polarizations. This states that the averaged sum of the photon's velocity shift for physical polarization equals $-\frac{(4 b+8 c)}{m_{\mathrm{e}}^{2}} R_{a b} k^{a} k^{b}$ [6]. We put $R_{a b}=\Lambda g_{a b}$. At one-loop order we may take $k^{2}=0$ on the right hand side. This means on the average there is no velocity shift for photons in $\Lambda$-vacuum spacetimes. This corresponds to the earlier identically vanishing results $[1,8]$. For our present case any of our non-vanishing results occurs like $1 \pm \epsilon$ for any set of two physical polarizations [Eqs. $(8-11,19,20)]$. Hence our result is consistent with the polarization sum rule.

It is clear that for any other $\Lambda$-vacuum spaces, the velocity shift, if it exists, should always be of the form $1 \pm \epsilon$, for any set of two physical polarizations.

Before we end, we shall briefly discuss now the extension of the above calculations in the off-equatorial plane. In that case we retain all the curvature components of (14). Due to this, two different physical polarizations mix with each other, unlike all the previous cases. For example, the equation for the azimuthal photons corresponding to the physical polarizations $(a \sim m, n)$ become 


$$
\begin{aligned}
& k^{2}\left(1+\frac{2 b \Lambda}{m_{\mathrm{e}}^{2}}\right)(a \cdot m)-\frac{8 c}{m_{\mathrm{e}}^{2}}\left[A k_{1}^{2}+B k_{0}^{2}+2 C_{1} k_{0} k_{1}\right](a \cdot m) \\
& -\frac{24 c C_{2} k_{0} k_{1}}{m_{\mathrm{e}}^{2}}(a \cdot n)=0, \\
& k^{2}\left(1+\frac{2 b \Lambda}{m_{\mathrm{e}}^{2}}\right)(a \cdot n)+\frac{8 c}{m_{\mathrm{e}}^{2}}\left[A k_{1}^{2}+B k_{0}^{2}+2 C_{1} k_{0} k_{1}\right](a \cdot n) \\
& -\frac{24 c C_{2} k_{0} k_{1}}{m_{\mathrm{e}}^{2}}(a \cdot m)=0,
\end{aligned}
$$

where $C_{1}=\frac{3 M a \sqrt{f} \sin \theta}{r^{4}}$ and $C_{2}=-\frac{3 M a \cos \theta}{r^{4}}$, and $A$ and $B$ are the same as earlier. In this case we need to solve the determinantal equation to get the modified dispersion relation. It is easy to check that the additional terms coming from the $C_{2}$ functions do not make any $\mathcal{O}(a)$-contribution. Also, in Eqs. (19) and (20), the factor $2 a$ is now replaced with $2 a \sin \theta$. This simplification certainly appears due to keeping terms linear in the rotation parameter. The $\sin \theta$ term represents the fact that the effect of rotation is vanishing along the axis, $\theta=0, \pi$, and maximum on the equatorial plane, $\theta=\pi / 2$.

\section{Summary and outlook}

Let us summarize the results now.

We have considered two physically interesting stationary $\Lambda$-vacuum spacetimes - the de Sitter cylindrical cosmic string and the Kerr-de Sitter case. For the cosmic string spacetime, we have found a shift for radial and orbital photons [Eqs. (8-11)], but no shift for axial photons. The vanishing of the velocity shift along the axis can be understood as the Lorentz symmetry in the ' $t-z$ ' plane of the cosmic string spacetime [Eq. (3)].

For the Kerr-de Sitter universe, we have only retained terms linear in the rotation parameter $a$, which should be the case far away from the source, where $\Lambda$ can play a prominent role. For the azimuthal photons, we have found a nonvanishing contribution from $\Lambda$ [Eqs. $(19,20)]$.

We note that for the two different spacetimes, $\Lambda$ contributes to the velocity shift in two qualitatively very different ways. However, all are consistent with the polarization sum rule. Investigation of this effect for gravitons seems interesting; this we hope to address in a future work.

Acknowledgments This research is implemented under the "ARISTEIA II" Action of the Operational Program "Education and Lifelong Learning" and is co-funded by the European Social Fund (ESF) and Greek National Resources.

Open Access This article is distributed under the terms of the Creative Commons Attribution 4.0 International License (http://creativecomm ons.org/licenses/by/4.0/), which permits unrestricted use, distribution, and reproduction in any medium, provided you give appropriate credit to the original author(s) and the source, provide a link to the Creative Commons license, and indicate if changes were made.

Funded by $\mathrm{SCOAP}^{3}$.

\section{References}

1. I.T. Drummond, S.J. Hathrell, Phys. Rev. D 22, 343 (1980)

2. T.J. Hollowood, G.M. Shore, Int. J. Mod. Phys. D 21, 1241003 (2012)

3. R.D. Daniels, G.M. Shore, Nucl. Phys. B 425, 634 (1994)

4. I.B. Khriplovich, Phys. Lett. B 346, 251 (1995)

5. R.D. Daniels, G.M. Shore, Phys. Lett. B 367, 75 (1996)

6. G.M. Shore, Nucl. Phys. B 460, 379 (1996)

7. H.T. Cho, Phys. Rev. D 56, 6416 (1997)

8. R.G. Cai, Nucl. Phys. B 524, 639 (1998)

9. G.M. Shore, Contemp. Phys. 44, 503 (2003)

10. G.M. Shore, Nucl. Phys. B 778, 219 (2007)

11. T.J. Hollowood, G.M. Shore, JHEP 0812, 091 (2008)

12. T.J. Hollowood, G.M. Shore. arXiv:1006.1238 [hep-th]

13. T.J. Hollowood, G.M. Shore, Phys. Lett. B 691, 279 (2010)

14. T.J. Hollowood, G.M. Shore, JHEP 1202, 120 (2012)

15. Y. Ohkuwa, Prog. Theor. Phys. 65, 1058 (1981)

16. R. Stanley, T.J. Hollowood, JHEP 1110, 096 (2011)

17. J.I. Latorre, P. Pascual, R. Tarrach, Nucl. Phys. B 437, 60 (1995)

18. W. Dittrich, H. Gies, Phys. Rev. D 58, 025004 (1998)

19. A.G. Riess et al. [Supernova Search Team Collaboration], Astron. J. 116, 1009 (1998)

20. S. Perlmutter et al. [Supernova Cosmology Project Collaboration], Astrophys. J. 517, 565 (1999)

21. Q. Tian, Phys. Rev. D 33, 3549 (1986)

22. S. Bhattacharya, A. Lahiri, Phys. Rev. D 78, 065028 (2008)

23. B. Carter, Commun. Math. Phys. 10, 280 (1968)

24. S. Chandrasekhar, The Mathematical Theory of Black Holes (Clarendon, Oxford, 1985) 